\title{
Use of TPS Software for Studying Fluctuating Asymmetry in Flowers
}

\author{
S. G. Baranov and D. E. Gavrikov
}

\begin{abstract}
The purpose of this study was to quantify the shape and compare fluctuating asymmetry (FA), as a random deviations from perfect bilateral symmetry of flowers of five species of plant. The shape analysis was based on the Procrustes method. The aligned shapes were used and treated in TPS series tpsDig, tpsUtil and tpsRegr. The first task was to test the index of fluctuating asymmetry using a 2 way ANOVA of homologous points to identify the most suitable species for testing developmental stability in unfavourable environments. The second task included the analysis of the homologous bilaterally-symmetrical distances in comparison to the index of fluctuating asymmetry. Fluctuating asymmetry showed a small fraction of the variability in flower shape. One species (Digitalis purpurea $L$.) showed the highest level of FA indicating deviation from developmental stability. The index of FA data on homologous landmarks corresponded to the data on fluctuating asymmetry index of distances.
\end{abstract}

Index Terms-Fluctuating asymmetry, shape analysis, procrustes method.

\section{INTRODUCTION}

One of the kinds of asymmetry is fluctuating asymmetry (i.e., a random deviation from perfect symmetry). It is interpreted as a measure of the developmental stability of the organisms in a population. The influence of fluctuating asymmetry on the plants described in many guides and scientific papers. Most of the work represents a deviation from the strict bilateral asymmetry (FA) as a result of the stress of the environment [1], [2].

A large body of work on the evaluation of FA employs the use of leaves with bilateral asymmetry. Only a few field studies have focused on FA in flowers. Insect-pollinated flowering plants have shown that bumblebees tend to collect nectar from symmetric flowers [3]. Some other studies show a correlation between FA in flowers and FA in leaf blades [4]. For example, in South Africa, FA of the leaves and flowers of about 15 representatives of different families have been analysed [5].

The modern approach to defining fluctuating asymmetry is based on the quantification of the properties of the form of the body or its parts. In this case, the deviation of pre-selected points from the corresponding points of some average models is taken into account. Comparing the deviations from the left and right sides permit conclusions about asymmetry including fluctuating asymmetry [6], [7].

Most plants have bilaterally symmetrical flowers, as well

Manuscript received December 7, 2012; revised February 28, 2013.

S. G. Baranov is with Vladimir State University, Russia. Professor University UDOM, Tanzania (e-mail: bar.serg58@gmail.com).

D. E. Gavrikov is wth e-Learning Department, East-Siberian State Academy of Education, Russia (e-mail: dega.irk@gmail.com). as leaf blades. Flowers with radial asymmetry lie outside the scope of the study of developmental stability and require a special approach.

It is logical to assume that deviation from symmetry in the high or low value at the same time leads to a change in form that differs from perfect symmetry and shape.

However, the evaluation of the FA index is not an easy task. One reason lies in the difficulty in selecting suitable symmetry points (landmarks) and their reliability for detecting FA. There are various approaches to describing FA, for example, including comparing the area of the two halves of the leaf blade.

The objective of the present work was to compare two indexes of FA in five plant species by studying flower form. In our study the analysis of flower shape using two indexes is based on the so-called Procrustes method. The first index is based on the coordinates of homologous landmarks, and the second index is based on the distance between homologous land marks on the left side and the right side.

In the base of the programs TPS family there is an aligning principle of all the points of interest to the user. The $(X Y)$ coordinates (in a two dimensional Cartesian coordinate system) are arranged around a zero point. The averaged model of a polygon is constructed first with known $(X Y)$ for each point.

The basic idea of the shape analysis is applying the possible configurations and determining the most appropriate configuration for all flower samples. It employs the method of least squares. Numerically, a form is analysed as the deviation in the variance between the points of the average shape (aligned centroid) and the corresponding points of real samples.

How is FA detected numerically? The definition of FA is any deviation from bilateral symmetry, usually quantified as numerically unequal differences between the absolute values (no signs) of the right and left sides. Thus, the index of FA can be determined from the difference of coordinate $(X Y)$ values between left and right homologous points. Statistical evaluation is determined using an Analysis of Variance (as a paired difference between left and right values of $X Y)(F$ test).

The task of the present study is to determine the fluctuating asymmetry of homologous points of inflorescences in five species of plants and identify the most suitable plant species for testing developmental stability. In doing so, two indexes of fluctuating asymmetry were also compared.

\section{MATERIALS AND METHODS}

\section{A. Site and Species}

Digital images of flowers were taken during the wet season 
(April-May, 2012) using a Panasonic DMC-FZ100 camera. The study area was about $2 \mathrm{~km}$ to the north-west of Dodoma town $\left(6^{\circ} 10^{\prime} 23^{\prime \prime} \mathrm{S} 35^{\circ} 44^{\prime} 31^{\prime \prime} \mathrm{E}\right)$ in the central part of United Republic of Tanzania. The site locality was typically woodland area described as forest-steppe subtropical zone. The elevation was $1500 \mathrm{~m}$ and total area about $5 \mathrm{~km}^{2}$.

The vegetation consisted of a steppe community of tall grasses and forbs. Anthropogenic factors included annual burning of dry vegetation, soil and degradation due to grazing and walking, litter and discarded building material.

The plant species used were common to Central Tanzania: Ipomea alba (f.Convolvulaceae Vent.), Coreopsis verticulata (f.Asteraceae, Compositae), Digitalis purpurea $L$. (f.Scrophulariaceae), Aspalathus capensis (Walp.) (f.Fabaceae) and Hibiscus engleri K.Schum (f.Malvaceae).

\section{MEASURING AND STATISTICS}

Detecting the differences in the flower form. In the software package TPS (J. Rohlf, 2010) factor "size" is supposed to reject for alignment as well as the rejection of outliers as points outside the confidence interval of the sample. JPG file format was used in the TPS program to determine the coordinates of landmarks for the next comparison between the shape of the sample and the overall average shape. The method of least squares was applied to obtain the aligned centroid. Deviation of the shape from the average geometric shape was detected by comparing the sum of variance of the centroid (average of the polygon) and the sum of variance of polygons in the sample. We used 60-80 digital images for each species of plant.

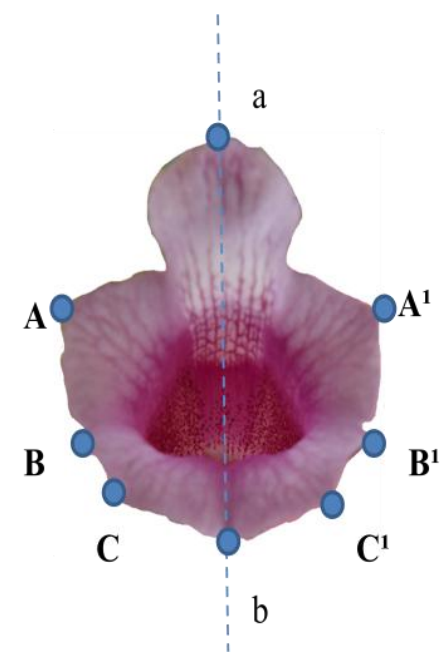

Fig. 1. Bilaterally-symmetrical landmarks and axis of bilateral symmetry (ab) of Digitalis purpurea $L$.

The criterion $F$ (F-Goodle), for description of the corresponding form of samples to the average centroid was used.

One real point was pre-defined on the $Y$-axis. The second point was the geometrical centre of the flower. Thus the assumption was that the centre of the centroid $(00)$ coincided with the centre of the inflorescence. Thus the two points defined the axis of bilateral symmetry (Fig. 1).

FA testing. The projection of homologous landmarks on the tangential space was used taking into account the angle to the zero point.
Thus, each point received new coordinates $(X Y)$ in the tangential space; the set of points was concentrated around the point (00).

The software PAST(ver.2.02) finds the distance between the left set and homologous right set of landmarks. This data was used to determine FA and its statistical significance using the Fisher criterion $(F)$.

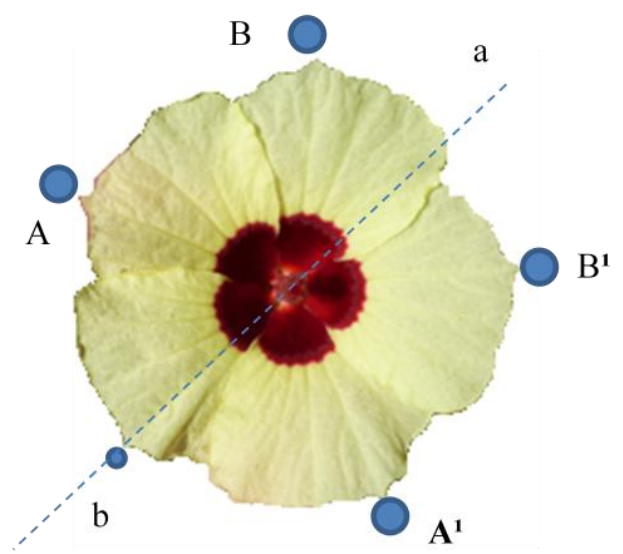

Fig. 2. Hibiscus engleri with radial asymmetry characteristics.

Previously, deviation of each pair of landmarks (from two to three pairs) was tested on the directional asymmetry (NA) and antisymmetry[7], [8], The two variables of the sample were tested for the mean difference from zero. Coordinates $(X Y)$ of left and right landmarks were tested. If the hypothesis that the difference between zero was rejected $(P<0)$, it indicated the presence of NA.

Antisymmetry was tested on tabulated data of kurtosis [8]. If the value of kurtosis of the difference $\left(X Y_{r}-X Y_{l}\right)$ is higher than the tabulated values, it signalled the presence of antisymmetry, i.e. the presence of significant deviation from the normal distribution.

To detect the components of variances and deviations, a two-way ANOVA was used.

The aim of this analysis was:

a) to detect individual variation in the sample;

b) to detect statistical significance of factor "side" (left and right) and make a conclusion about the presence of directional asymmetry;

c) to find measurable error affecting FA value.

FA was calculated using the formula:

$$
\sigma^{2}=\left(M S_{i s}-M S_{m}\right) / M,
$$

where:

$\sigma^{2}-$ non-directional asymmetry variance after removing measurement error;

$M S_{i}$ - mean square interaction "side" and "individuals";

$M S_{m}$ - mean square measurement error;

$M$ - amount of measurements (three repeats).

\section{RESUlts}

\section{A. Shape Testing}

A small deviation in shape from the average aligned model was detected in hibiscus (F-Goodle $=8.68 ; d f=192 ; P=$ $0.000)$. For other flowers $F$ criteria were higher (14.6-26.7; $d f$ 
$=192 ; \mathrm{P}=0.000)$. Thus variation in flower shape was low within each species.

\section{B. FA Testing}

Index of FA using the coordinates of the tangential space was determined by including the product of the coordinates of the left and right homologous points in formula (1) which provided the final result of the two-way ANOVA (Table I).

Fisher's exact test was used to determine statistical significance of null hypothesis of no difference between variance products $(X Y)$ left and right side.

For the index of FA using homologous distances, the segments joining homologous points on the left and the right sides of flowers were used, also applying the $F$ criterion.

The interaction of both factors "side" and "individuals" showed a high value of mean square and a low value of mean square measurement error that suggested the presence of FA. Thus, the $F$ value suggested significant FA for some species. Only one species among the five (Digitalis purpurea) showed absence of DA (factor "side" was insignificant).

TABLE I: RESULTS OF 2 WAY ANOVA (FA AND DA)

\begin{tabular}{|c|c|c|c|c|}
\hline \multirow[t]{2}{*}{ Species } & \multicolumn{2}{|c|}{ FA landmarks } & \multicolumn{2}{|c|}{ FA distance } \\
\hline & Index & $F$ & Index & $F$ \\
\hline $\begin{array}{l}\text { Digitalis } \\
\text { purpurea L. }\end{array}$ & $\begin{array}{l}0.0004 \\
0.0000\end{array}$ & $\begin{array}{l}17.5 * * \\
17.2 * *\end{array}$ & $\begin{array}{l}0.0002 \\
0.0001\end{array}$ & $\begin{array}{l}20.9 * * \\
20.7 * *\end{array}$ \\
\hline $\begin{array}{l}\text { Aspalathus } \\
\text { capensis } \\
\text { (Walp.) }\end{array}$ & $\begin{array}{l}0.001 \\
0.001\end{array}$ & $\begin{array}{c}19.80 ; D A \\
29.47 ; D A\end{array}$ & $\begin{array}{l}n s \\
n s\end{array}$ & $\begin{array}{l}n s \\
n s\end{array}$ \\
\hline $\begin{array}{l}\text { Coreopsis } \\
\text { verticulata }\end{array}$ & 0.0004 & $0.444 * ; D A$ & $n s$ & $n s$ \\
\hline $\begin{array}{l}\text { Hibiscus } \\
\text { engleri, } \\
\text { Ipomea alba }\end{array}$ & $n s$ & $n s ; D A$ & $n s$ & $n s$ \\
\hline
\end{tabular}

Note:

$n s-$ statistically insignificant $(P>0.05)$;

** $-P<0.0001$;

$*-P<0.001$

Thus Digitalis showed significant FA result in both analyses for landmarks and for distances.

A significant index of FA was not detected for every pair of landmarks. Some pairs of landmarks showed FA, but in combination with other landmarks, the common FA index was insignificant (Coreopsis verticulata). The reasons were the presence of DA or insignificant index of FA.

\section{Testing of Directional Asymmetry and Antisymmetry}

Directional asymmetry was present $(P<0.05$; two-sample $t$-test with different variances) for almost all species. The result corresponded to the data of 2 way ANOVA (significant factor "side").

The presence of antisymmetry and deviation from normality was evident for all species. The value $\left(X Y_{r}-X Y_{l}\right)$ showed high kurtosis $(k>7.54)$ typical for leptokurtic distributions. The kurtosis value was higher than tabulated (n $=80-90 ; \alpha=0.05$ ).

The flowers with trend to radial asymmetry clearly showed directional asymmetry or undetectable FA, for example Hibiscus (Fig. 2) and Ipomea. Even strictly bilaterally symmetrical Aspalathus capensis (f.Legumiacea) also showed DA. Within one species, depending on landmarks, directional asymmetry on the right side or the left side was observed.

\section{DISCUSSION AND CONCLUSION}

The experience in the use of the TPS (using the sequence tpsDig - tpsUtil - tpsRegr) software package showed that:

1) The program is quick and easy to use and allows for a variety of homologous bilaterally symmetrical landmarks;

2) Fluctuating asymmetry, as a slight deviation from symmetry, is a small fraction of the variability of flower shape. In this study, FA did not play a significant part in explaining variation in flower shape for all five species.

3) Digitalis purpurea showed a significant level of fluctuating asymmetry using both approaches (landmark and distance coordinates) and may prove to be a useful bioindicator of stressful factors in subtropical areas in Tanzania.

\section{ACKNOWLEDGMENT}

We are grateful to Dr S. Ratnayeke for editing.

\section{REFERENCES}

[1] D. C. Freeman, J. H. Graham, J. M. Emlen, M. A. Tracy, R. A. Hough, C. L. Alados, and J. Escós, "Plant developmental instability: New measures, applications, and regulation," in Developmental instability: causes and consequences, Polak M., ed., New York: Oxford University Press, 2003, pp. 367-386.

[2] S. B. Samuelsson and S. Andersson, "The effect of nutrient stress on developmental instability in leaves of Acer platanoides (Aceraceae) and Betula pendula (Betulaceae)," American Journal of Botany, vol. 90 , no. 8, pp. $1107-1112,2003$

[3] A. P. Møller, "Bumblebee preference for symmetrical flowers," in Proc. the National Academy of Science USA, no. 92, 1995, pp. 2288-2292.

[4] A. P. Møller and M. Eriksson, "Patterns of fluctuating asymmetry in flowers: implications for sexual selection in plants," J. Evol. Biol., no. 7, pp. 97-113, 1994.

[5] M. D. Jennions, "The allometry of fluctuating asymmetry in southern African plants: flowers and leaves," Biological Journal of the Linnean Society, no. 59, pp. 127-142, 1996.

[6] C. P. Klingenberg, M. Barluenga, and A. Meyer. "Shape analysis of symmetric structures: quantifying variation among individuals and asymmetry," Evolution, vol. 56, pp. 1909-1920, 2002.

[7] C. P. Klingenberg and G. S. McIntyre. "Geometric morphometrics of developmental instability: analyzing patterns of fluctuating asymmetry with Procrustes methods," Evolution, vol. 52, pp. 1363-1375, 1998.

[8] A. R. Palmer and C. Strobeck. "Fluctuating asymmetry analysis revisited," in Developmental instability: causes and consequences, M. Polak, ed., New York: Oxford University Press, 2003, pp. 279-319.

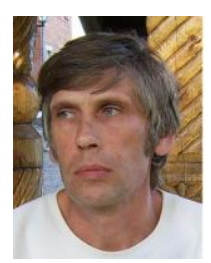

Sergey Baranov was born on 25th June 1960 in Vladimir, Russia. My educational background is as follows: I graduated from Vladimir State University (VSU) with a Russian Masters in Science, namely Biology and Chemistry in 1982. Thereafter, I attended the 'All-Russian Research Institute for Animal Health' in Vladimir, Russia and trained for a $\mathrm{PhD}$ in Biology in 1989. I, Dr. Sergey Gennadevich Baranov was a docent and Chair of Botany at the Natural-Geographical faculty of VSU in Vladimir, Russia in 1996. I became a member of ISEE (International Society for Ecological Economics) in 2001 and I have been a Senior lecturer at UDOM University, College of Natural Sciences and Mathematics inTanzania from 2010-present day. My basic publications include: S. G. Baranov and A. V. Maleev, "To the use of harmonic analysis in phenogenetic study," Electronic mathematical and medical-biological journal, vol.8, no.3, pp.1-9, 2009; D. E. Gavrikov and S. G. Baranov, "The developmental stability estimation technique on birches example (Betula pendula)," Bulletin OSSC SB RAMS, no. 2, pp. 13-17, 2006; S. G. Baranov, 
"Ecology of bacteria and virus," Victory book printers, Dodoma, 2012, 78 p. DR. S.G. BARANOV, DOCENT Vladimir State University (VSU). My research interests are: Fluctuating asymmetry of plants as an indicator of environmental stress, Geometric morphometrics, Biostatistics, Biomonitoring and Bioindication. My present professional occupation is lecturing on: Ecology, Parasitology and Microbiology. I was awarded first prize at an open contest of «Green-PIK» JSC, Consortium of Earthworm for the work, "Study of the effect of vermicompost on stability of the pumpkin by analyzing the leaf blades asymmetry" in Kovrov, Russia in 2005.

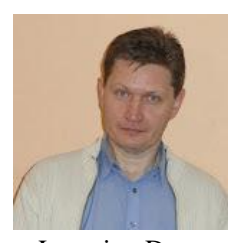

Dmitry Gavrikov was born on May 3, 1973, in Irkutsk, Russia. He graduated from Ecological Genetics Laboratory, Biological Research Institute at Irkutsk State University, Russia (ISU), as Post Graduate Student, PhD (biology), in 1998. He was Assistant Ecology and Nature Sciences Chair in 2002. Dr. Dmitry Eugenevich Gavrikov is Chief of e-Learning Department, East-Siberian State Academy of Education, Irkutsk, Russia (since 2006).

His present professional occupation is statistical analys biological data and ecological education. Research interests: ecological genetics; asymmetry of bilateral characters; environmental and genetic mechanisms of developmental stability. 